九州大学学術情報リポジトリ

Kyushu University Institutional Repository

\title{
Optimization of Ion Thruster Grids Using JIEDI Code with Genetic Algorithm
}

Inoue, Atsumu

Department of Advanced Energy Engineering Science, Kyushu University

Yamamoto, Naoji

Department of Advanced Energy Engineering Science, Faculty of Engineering Science, Kyushu University

Nakamura, Yusuke

Department of Advanced Energy Engineering Science, Kyushu University

Nakano, Masakatsu

Tokyo Metropolitan College of Industrial Technology

http://hdl. handle. net/2324/4377911

出版情報: Transactions of the Japan Society for Aeronautical and Space Sciences, Aerospace Technology Japan. 19 (1)，pp.75-80，2021-01-04. 日本航空宇宙学会

バージョン:

権利関係: (c) 2021 The Japan Society for Aeronautical and Space Sciences 


\title{
Optimization of Ion Thruster Grids Using JIEDI Code with Genetic Algorithm
}

\author{
By Atsumu Inoue, ${ }^{1)}$ Naoji YAmamoto, ${ }^{1)}$ Yusuke NAKAmura,,${ }^{1)}$ and Masakatsu NAKANO ${ }^{2)}$ \\ 1) Department of Advanced Energy Engineering Science, Kyushu University, Kasuga, Japan \\ 2) Tokyo Metropolitan College of Industrial Technology, Tokyo, Japan
}

(Received July 12th, 2019)

\begin{abstract}
The grid design of ion thrusters is currently based on trial and error development and durability tests. These methods make it difficult to rapidly develop optimal thrusters for diverse missions. As a way to address this problem, this paper provides a method of designing grids using numerical simulation with a genetic algorithm. Screen grid thickness, screen grid hole diameter, accel grid thickness, accel grid hole diameter, accel grid potential, and grid gap are optimized for long-life grids. The JIEDI tool is a powerful tool to design grid systems; it is used here as a fitness function to evaluate the lifetime of the grid in an ion thruster. As a result of the optimization, the lifetime of the model developed using the genetic algorithm is about 6.5 times longer than that of the experimental model. The optimization of grid parameters using the Genetic Algorithm and the JIEDI tool shows promise for future design tasks.
\end{abstract}

Key Words: Ion Engine, Grid Erosion, JIEDI Tool, Genetic Algorithm, Lifetime Assessment

Nomenclature
$\begin{array}{cll}\mathrm{d} & : & \text { hole diameter, } \mathrm{mm} \\ f & : & \text { fitness function } \\ k & : & \text { constant } \\ l_{I} & : & \text { gap between the grids, } \mathrm{mm} \\ P & : & \text { probability being selected } \\ \mathrm{t} & : & \text { thickness, } \mathrm{mm} \\ u & : & \text { random number } \\ \mathrm{V} & : & \text { potential, } \mathrm{V} \\ x & : & \text { value of a parameter } \\ \alpha & : & \text { expansion rate in crossover } \\ \eta & : & \text { proportion }\end{array}$

Subscripts

$\begin{array}{lll}\mathrm{a} & : & \text { accel grid } \\ i & : & \text { individual number } \\ \mathrm{S} & : & \text { screen grid }\end{array}$

\section{Introduction}

In recent years, space development has advanced rapidly with missions as diverse as planetary exploration and artificial satellites for commercial purposes. In this trend continues, various requirements will be imposed on ion thrusters, one of the promising electric propulsion systems. Design of the grids is very important in the development of ion thrusters, because the grid plays an essential role in accelerating ions, and greatly affects both the performance and the lifetime of the propulsion system.

Grid design involves a large number of interdependent parameters, ${ }^{1}$ generally using heuristic methods, trial and error, and durability tests. These methods, however, are expensive and time-consuming, ${ }^{2)}$ and the cost and time are expected to increase with the required lifetime of thrusters. This makes it difficult to develop ion thrusters in line with the fast-changing trends of the times.

The present research investigates grid design by numerical simulation with machine learning. Six parameters: screen grid thickness, screen grid hole diameter, accel grid thickness, accel grid hole diameter, accel grid potential, and grid gap, are optimized using a genetic algorithm (GA) to design a long-life grid. One problem in grid design is how to reconcile a large number of optimal parameters; the cost of calculating optimal parameters among the huge number of possible combinations is likely to be high unless the algorithm has learning ability. Therefore, GA, which is a kind of machine learning, was selected as the optimization method. The details of the choice of GA are discussed in Section 3. The lifetime of the developed ion thruster is calculated using the JAXA Ion Engine Development Initiative (JIEDI) tool. This tool is introduced in Section 2.

\section{Numerical Simulation}

The JIEDI tool developed by JAXA is a numerical simulation code to evaluate the lifetime of a grid in an ion thruster. The flow of charged particles is treated as a current beam, which reduces calculation cost. ${ }^{3)}$ Reproducibility has been tested through comparison of experimental and simulation results, and agreement has been deemed sufficient. ${ }^{4)}$

A two-gridded ion thruster is analyzed in this research, and the analysis area is $1 / 12$ of one grid hole, assuming the symmetry of the hole arrangement as shown in Fig. 1. The analysis model and six parameters are shown in Fig. 2. Ions are accelerated in the positive $\mathrm{z}$-direction. Other parameters and the operating conditions are shown in Table 1. 


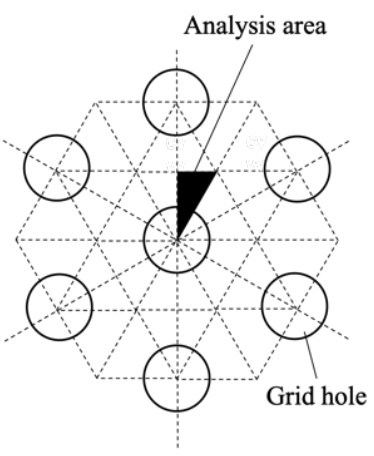

Fig. 1. The symmetry of the hole arrangement.

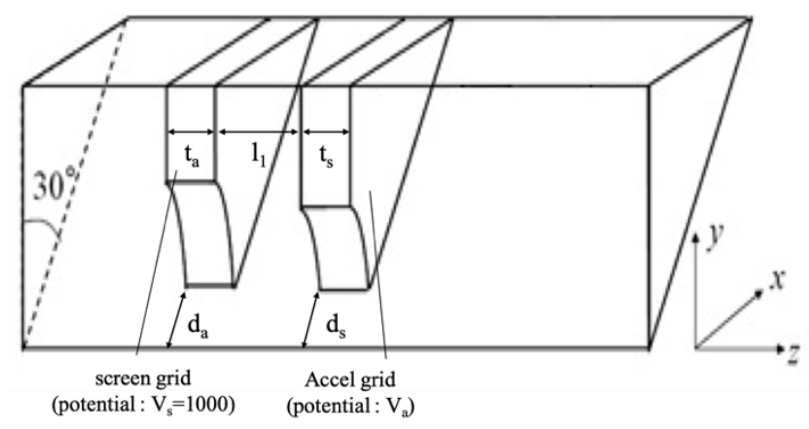

Fig. 2. Schematic of the two-grid system analyzed here.

Table 1. Parameters and operating conditions.

\begin{tabular}{ll}
\hline Grid material & $\mathrm{CC}$ \\
Propellant & $\mathrm{Xe}$ \\
Screen grid potential: Vs & $1000 \mathrm{~V}$ \\
Open area fraction of screen grid & 0.58 \\
Temperature of grid surface & $403 \mathrm{~K}$ \\
Beam current per hole & $1.31 \times 10^{-1} \mathrm{~mA}$ \\
Propellant utilization efficiency & 0.85 \\
Sputtering model & Williams \\
\hline
\end{tabular}

\section{Genetic Algorithm}

\subsection{Introduction of GA}

In this research, GA is used to optimize the parameters of the grid. GA was proposed by John Holland in 1975. It is an optimization method modeled on the mechanism of natural selection. ${ }^{5)}$ In nature, each species, or even individuals in the same species, compete with each other for food, water, and habitat. Through these competitions, the winners thrive, and the losers become extinct. In other words, the species that is most suitable for the environment survives. GA searches for an optimum solution via a similar mechanism. ${ }^{6)}$ The process is explained in the next section. GA was chosen for the optimization of grid parameters because it is necessary to have a learning ability rather than just an optimization method. GA can be used for the optimization of the parameters only if the fitness function is decided. Therefore, it can be applied to optimization under different conditions simply by changing the fitness function.

\subsection{Advantages}

The advantages of GA are as follows.

1. It can search for optimal values even on a multimodal function by a multi-point search.

2. It is a direct search by a fitness function.

3. It can find multiple optimal values simultaneously in a single search.

A multi-point search makes it possible to easily escape from local solutions. Thanks to direct search, it can be used for complex problems that are difficult to solve analytically, and problems whose properties have not yet been clarified, as long as the problem is evaluated by a fitness function. Because GA has high robustness to search domain, it is effective in problems that would challenge conventional search algorithms such as random search and hill climbing methods. ${ }^{7)}$ Moreover, it is compatible with parallelization, maximizing computer performance.

\subsection{Process}

Figure 3 shows a flowchart illustrating the GA procedure. The GA can be divided into six steps, each of which is described below briefly.

1. Initial population: Generation of individuals with random parameters (usually called genes). The relationship between individuals and genes is showed in Fig. 4 graphically.

2. Fitness evaluation: Evaluation of each individual. Each individual is given a fitness score.

3. Selection: Selecting parents based on fitness score.

4. Crossover: Producing child individuals in the next generation from parent individuals.

5. Mutation: Addition of small changes to individuals generated in crossover with low probability.

6. Convergence judgment: Convergence of the optimization is evaluated depending on the predetermined convergence conditions, and the optimal parameters are determined. If the convergence condition is not satisfied, the steps 2-5 are repeated. ${ }^{8)}$

By repeating the process of generating the next generation from individuals with high score evaluated by fitness function, individuals approach the one with desirable characteristics (genes). Each process is described in detail in the following sections. Table 2 summarizes the methods of each process and the conditions used in the present study.

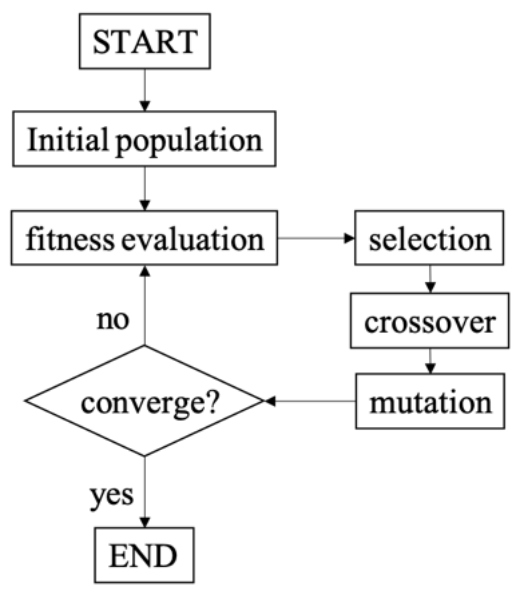

Fig. 3. GA procedure. 
Table 2. Summary of methods and conditions for GA.

\begin{tabular}{|c|c|}
\hline The size of population & 8 \\
\hline The number of genes & 6 \\
\hline Encoding & Real encode \\
\hline Selection & Roulette and elitism \\
\hline Crossover & $\begin{array}{l}\text { Expanded intermediate } \\
\text { crossover }\end{array}$ \\
\hline Mutation & BGA mutation \\
\hline Convergence condition & $\begin{array}{l}90 \% \text { of the genes become } \\
\text { equivalent to those of elite }\end{array}$ \\
\hline
\end{tabular}

\begin{tabular}{|c|c|c|c|c|c|c|}
\hline 0 & 0 & 0 & 1 & 1 & 1 & Individual 1 \\
\hline 1 & 1 & 0 & 1 & 0 & 1 & Individual 2 \\
\hline & & $\vdots$ & & & & $\vdots$ \\
\hline 1 & 0 & 0 & 1 & 1 & 0 & Individual 8 \\
\hline
\end{tabular}

Fig. 4. The relationship between the individuals and genes. The genes are expressed in binary for simplicity, but they are actually expressed in real value.

\subsection{Fitness function}

The fitness score represents an evaluation for each individual. The larger score the individual has, the higher the possibility that it will be selected and its genes continue to the next generation. In this research, the lifetime calculated by the JIEDI tool is directly used as fitness score in order to design a long-life grid. The end of the grid's lifetime is defined as the time when electrons begins to flow backward.

\subsection{Selection}

The selection process converges the population and eliminates individuals with low fitness score. There are various methods, but only the two methods used in this research are explained below. ${ }^{9}$ )

\subsubsection{Roulette wheel selection}

This method was proposed by John Holland. ${ }^{5)}$ According to Eq. (1), the probability of each individual being selected is proportional to its fitness score. An example is shown in Table 3 and Fig. 5.

$$
P_{i}=\frac{f_{i}}{\Sigma f_{i}}
$$

Parents are selected at random by the probability calculated by Eq. (1). This means that individuals with higher fitness score are more likely to be selected. In addition, there is some small probability that individuals with lower fitness score will be selected.

Table 3. Example of Roulette wheel selection.

\begin{tabular}{ccc}
\hline & Fitness score $f_{i}$ & Probability $P_{i}[\%]$ \\
\hline 1 & 110 & 48 \\
2 & 55 & 24 \\
3 & 60 & 26 \\
4 & 5 & 2 \\
\hline
\end{tabular}

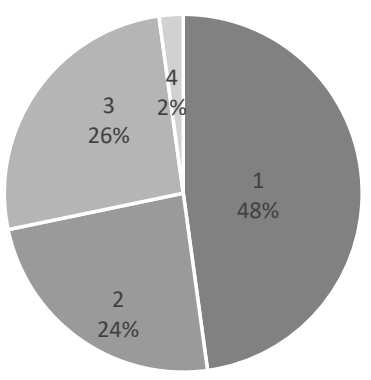

Fig. 5. Example of Roulette wheel selection.

\subsubsection{Elitism selection}

This is the simplest method. A predetermined number of individuals with the highest fitness score is left for the next generation. This method has the advantage that the maximum value of the fitness score does not decrease. However, the diversity of solutions is lost if an elite gene affects the next generation too much. In order to avoid this, the elite is usually added to the population if the elite has not been selected after other selection methods. ${ }^{10)}$ In this research, one elite individual is left for the next generation.

\subsection{Crossover}

Extended intermediate crossover, one of methods to generate an offspring by a linear combination of the parents, is used. Each parameter of each individual in the next generation is generated randomly from the extended range, which is $(1+2 \alpha)$ times larger than the range where the parent individuals are maximum and minimum, as shown in Fig. 6. $\alpha$ is set to 0.25 in the present study.

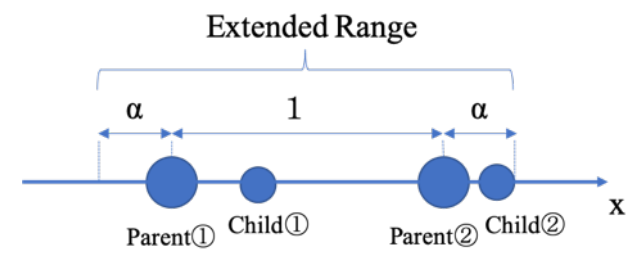

Fig. 6. Extended intermediate crossover.

\subsection{Mutation}

Mutation maintains the diversity of the population by expanding the search space. This is an important process because it prevents the search from falling into local solutions. In this research, BGA mutation is used as a mutation operator. ${ }^{11)}$ The parameter after the mutation $x_{i}^{\prime}$ is calculated according to

$$
x_{i}^{\prime}=x_{i} \pm 0.05 \cdot 2^{-u k},
$$

where $x_{\mathrm{i}}$ is the parameter before the mutation, $0 \leq u \leq 1$ and $k=8$. In this method, it does not need to manually adjust the mutation range even if the range of parameter is changed. Thus, this method is robust.

\subsection{Convergence condition}

Convergence is judged based on predetermined conditions. Examples of conditions could include:

1. An average or maximum of fitness score exceeds a predetermined criterion. 
2. Maximum fitness score does not increase during a set number of generations.

3. A predetermined number of iterations is executed. ${ }^{12)}$

In the first case, the maximum value of the fitness score must be defined in advance. If this estimated maximum value is too large, the calculation will not converge, and if it is too small, a better value cannot be found. The calculation in the second case ends when the maximum of the fitness score ceases to update even once. In the third case, the calculation cost can increase in some cases. In the present research, $\eta$ is defined as the proportion of the number of parameters equivalent to those in the elite individual in the total number of parameters in the generation, and the convergence condition is satisfied when $\eta$ exceeds $90 \%$

\section{Results}

Six parameters are optimized within the ranges shown in Table 4. These ranges are determined based on the values of an ion thruster used in the laboratory (experimental model). ${ }^{13)}$ In the selection, one individual is selected with Elitism and the parent individuals are selected with roulette wheel selection. After that, the seven individuals are generated from parent individuals in the crossover and the mutation applied to these individuals. The next generation consists of these seven individuals and elite individuals. As shown in Fig. 7, $\eta$ reaches $91.7 \%$, and the convergence condition is satisfied in the 10th generation. The numerical cost of this process was 48 hours. The result of optimization is shown in the Table 5. The result of evaluation of the experimental model by the JIEDI tool is also shown.

Table 4. The ranges of six parameters

\begin{tabular}{lccc}
\hline Parameter & Symbol & Range & Unit \\
\hline $\begin{array}{l}\text { Screen grid } \\
\text { thickness }\end{array}$ & $\mathrm{t}_{\mathrm{s}}$ & $0.10-0.50$ & {$[\mathrm{~mm}]$} \\
$\begin{array}{l}\text { Screen grid } \\
\text { hole diameter }\end{array}$ & $\mathrm{d}_{\mathrm{s}}$ & $1.00-1.40$ & {$[\mathrm{~mm}]$} \\
$\begin{array}{l}\text { Grid gap } \\
\begin{array}{l}\text { Accel grid } \\
\text { thickness }\end{array}\end{array}$ & $\mathrm{l}_{1}$ & $0.10-0.50$ & {$[\mathrm{~mm}]$} \\
$\begin{array}{l}\text { Accel grid } \\
\text { hole diameter }\end{array}$ & $\mathrm{t}_{\mathrm{a}}$ & $0.10-0.50$ & {$[\mathrm{~mm}]$} \\
$\begin{array}{l}\text { Accel grid } \\
\text { potential }\end{array}$ & $\mathrm{d}_{\mathrm{a}}$ & $0.50-0.90$ & {$[\mathrm{~mm}]$} \\
\hline
\end{tabular}

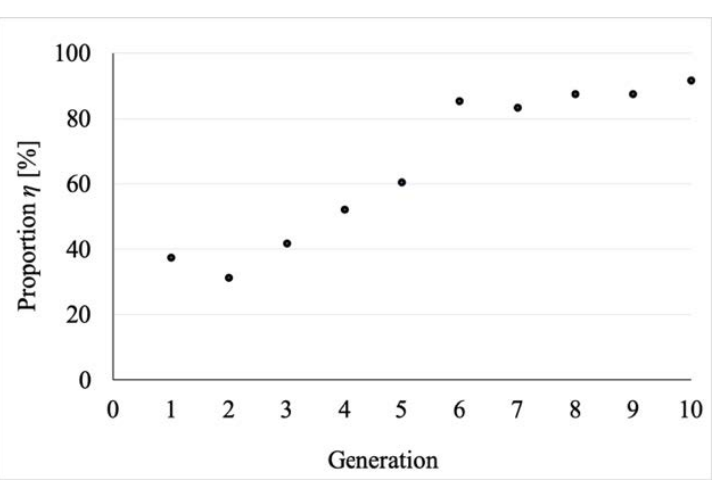

Fig. 7. The proportion $\eta$ of each generation.
Table 5. Parameters of experimental model and GA model.

\begin{tabular}{lccc}
\hline Parameter & $\begin{array}{c}\text { Experimental } \\
\text { model }\end{array}$ & $\begin{array}{c}\text { GA } \\
\text { model }\end{array}$ & Unit \\
\hline $\begin{array}{l}\text { Screen grid } \\
\text { thickness }\end{array}$ & 0.30 & 0.38 & {$[\mathrm{~mm}]$} \\
$\begin{array}{l}\text { Screen grid } \\
\text { hole diameter }\end{array}$ & 1.20 & 1.16 & {$[\mathrm{~mm}]$} \\
$\begin{array}{l}\text { Grid gap } \\
\begin{array}{l}\text { Accel grid } \\
\text { thickness }\end{array}\end{array}$ & 0.20 & 0.21 & {$[\mathrm{~mm}]$} \\
$\begin{array}{l}\text { Accel grid } \\
\text { hole diameter }\end{array}$ & 0.30 & 0.37 & {$[\mathrm{~mm}]$} \\
$\begin{array}{l}\text { Accel grid } \\
\text { potential }\end{array}$ & -150 & 0.53 & {$[\mathrm{~mm}]$} \\
\hline Lifetime & 35055 & -136 & {$[\mathrm{~V}]$} \\
\hline
\end{tabular}

\section{Discussion}

The grid shapes before and after operation are estimated for the experimental model using the JIEDI tool, as shown in Fig. 8, and for the GA-designed model as shown in Fig. 10. The highly eroded area is represented in red, while the low-erosion area is shown in blue. Figures 8 and 10 show that the accel grids in both models are eroded and hole diameters widen towards the end after operation. According to Table 5, the lifetime of the GA model is about 6.5 times longer than that of the experimental model. This means that the optimization of the parameters by GA is successful.

The accel grid hole diameter and the accel grid potential show large differences between the two models. Because of the higher accel grid potential, the energy of the charge exchange ions from downstream is reduced when they collide with the accel grid. Figure 8(c) shows that the downstream surface of the accel grid in the experimental model is eroded into a specific shape pattern called "pits and grooves" by the charge exchange ions, while no such pattern is seen in the GA model in Fig. 10(c). ${ }^{14)}$ This demonstrates that the higher potential has an effect on how the accel grid are eroded. In addition, the grid shape transition is different between the two models due to the smaller hole diameter of the accel grid. The beam goes through the grid hole from the beginning with experimental model. On the other hand, the accel grid with GA model is severely eroded due to the small grid hole initially. However, the shape of the hole with GA model is eroded according to the ion beam trajectory three-dimensionally. Therefore, the ion beam gradually stops colliding with the grid during the operation time although the initial spatter amount is large. This difference of the erosion process is shown in Fig. 12. A method of machining the grid hole into an optimum shape by the irradiation with ion beam has been studied, and high propellant utilization efficiency can be achieved after machining. ${ }^{15)}$ In this research, because only the lifetime is used for the evaluation, GA found the grid model close to the one designed by this method. Furthermore, the shape of the potential is maintained even when the grid hole diameter gets larger because the accel grid thickness with GA model becomes thicker than with experimental model. This is the main factor in achieving a longer grid lifetime. 

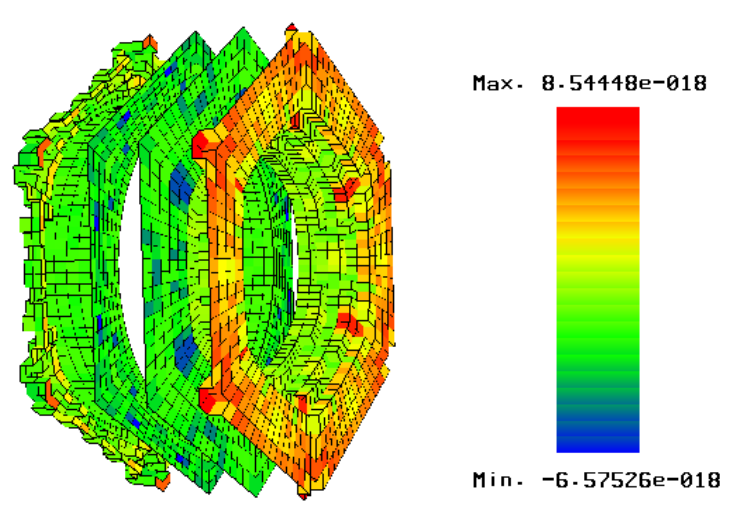

(a) Overview of the grid after operation.
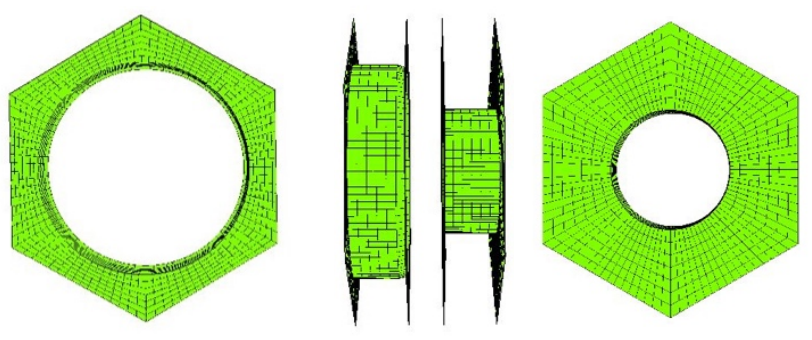

Upstream surface of screen grid

Side surface

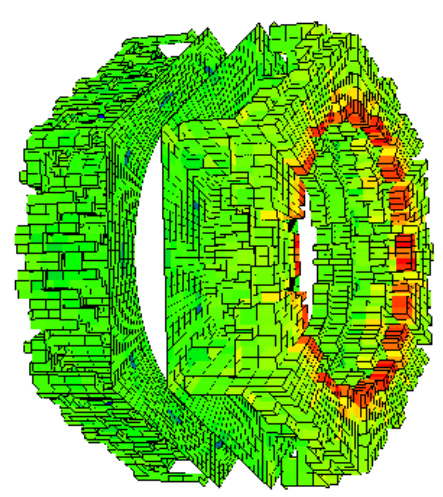

Max. $6 \cdot 33716 e-018$

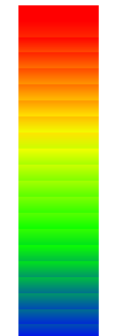

Min. $-4 \cdot 80912 e-018$

(a) Overview of the grid after operation.

(b) Each grid before operation.
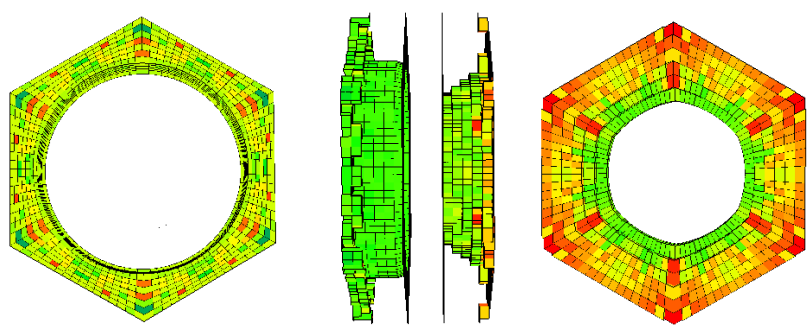

Upstream surface of screen grid Side surface

Downstream surface of accel grid

(c) Each grid after operation.

Fig. 8. Erosion rate of experimental model.

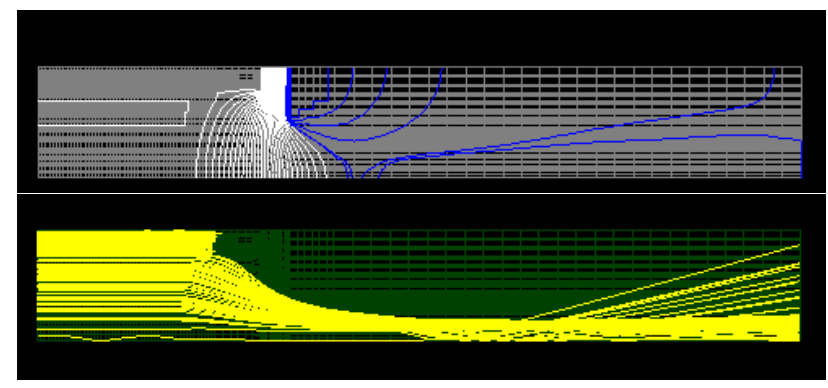

Fig. 9. Potential distribution (top) and ion beam trajectory (bottom) at the end of life with experimental model.

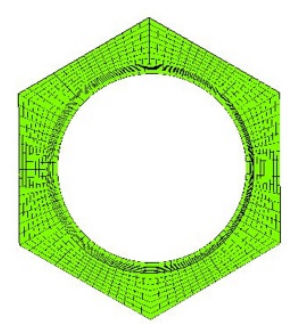

Upstream surface of screen grid

Side surface

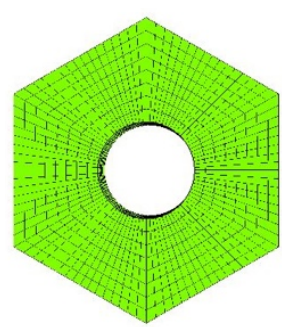

Downstream surface of accel grid (b) Each grid before operation.
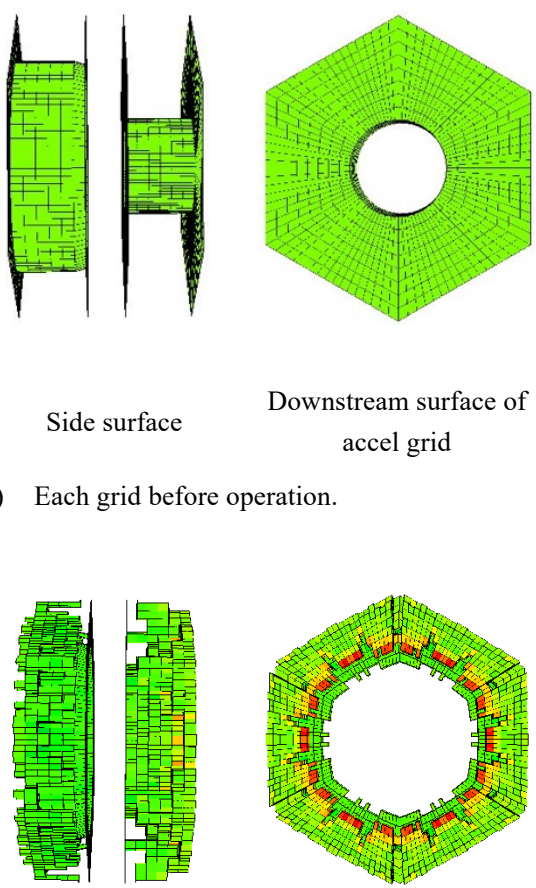

Upstream surface of screen grid

Side surface

Downstream surface of accel grid

(c) Each grid after operation.

Fig. 10. Erosion rate with GA model.

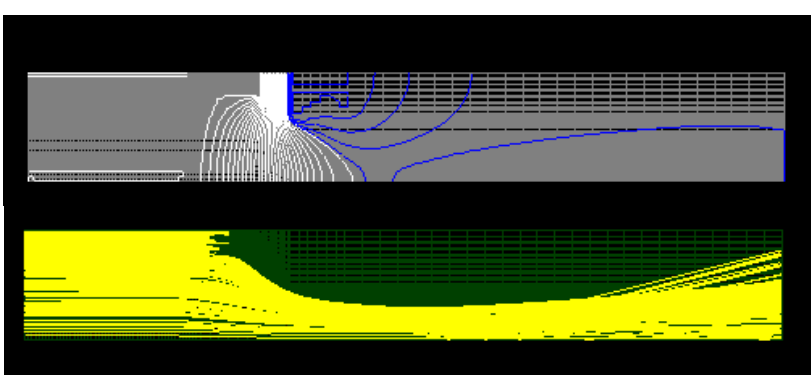

Fig. 11. Potential distribution (top) and ion beam trajectory (bottom) at the end of life with GA model. 


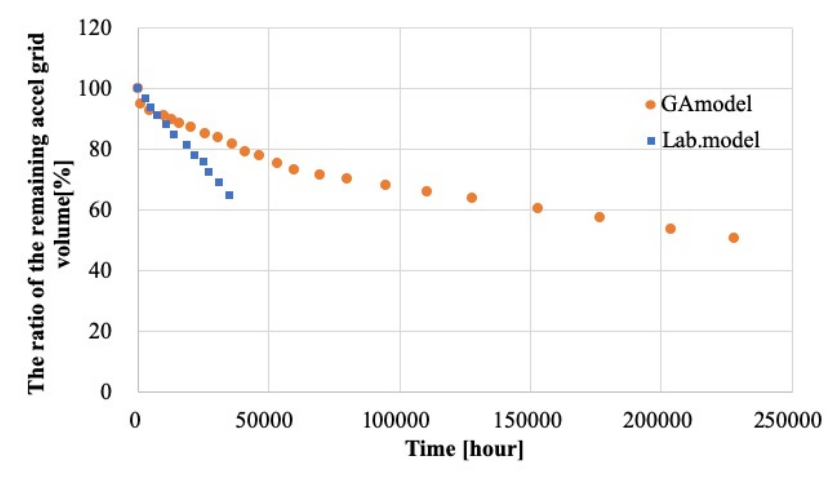

Fig. 12. The ratio of the remaining accel grid volume as a function of time.

The potential distribution and the ion beam trajectory at the end of life with the experimental and GA models are shown in Figs. 9 and 11, respectively. The potential distributions are almost identical in the two models. On the other hand, the ion beam forms a wider stream in the GA model than in the experimental model. As shown in the Table 6, however, the specific impulse is almost same in the two models.

Table 6. Specific impulse calculated using JIEDI tool.

\begin{tabular}{ccc}
\hline & $\begin{array}{c}\text { Experimental } \\
\text { model }\end{array}$ & $\begin{array}{c}\text { GA } \\
\text { model }\end{array}$ \\
\hline Specific impulse [s] & 3356 & 3352 \\
\hline
\end{tabular}

\section{Conclusion}

This research establishes a method of grid design in ion thrusters using a genetic algorithm to optimize grid parameters for a two-grid thruster for maximum lifetime. Six parameters grid gap, screen grid thickness, screen grid hole diameter, accel grid thickness, accel grid hole diameter, and accel grid voltage - are optimized. The optimized parameters were compared with those of the ion thruster actually used in the laboratory. The accel grid thickness is larger, the accel grid hole diameter is smaller, and the accel grid potential is higher than those of the experimental model. These changes make it possible to maintain the potential shape properly for a long time, and to improve the durability of the grids. As a result, the lifetime of the grid is predicted to be 6.5 times longer than that of the experimental model as calculated using the JAXA JIEDI tool. This result establishes that the optimization of grid parameters by numerical simulation with GA is useful and suggests an efficient method for ion thruster design.

\section{Acknowledgments}

This work was performed at the Space Plasma Laboratory of ISAS, JAXA. It was supported by the 2016-2018 JAXA Strategic Development Program and JSPS KAKENHI Grants Numbers JP16H04595, JP16K14506 and JP18H03815.

\section{References}

1) Hayakawa, Y., Kitamura, S., and Miyazaki, K.: A Theory for Designing Cusp Field Xenon Ion Thrusters, NAL-TM-751, 2000 (in Japanese).

2) Nishiyama, K., Hosoda, S., Ueno, K., Tsukizaki, R., and Kuninaka, H.: Development and Testing of the Hayabusa 2 Ion Engine System, Trans. JSASS Aerospace Tech. Japan, 14 (2016), pp. $\mathrm{Pb} \_131-\mathrm{Pb} \_140$

3) Nakano, M., Kajimura, Y., and Funaki, I.: Development of the JIEDI-1 tool: Improvements to the Grid Erosion Evaluation Code Developed in 2007, JAXA-RR-09-004, 2010, pp. 1-9 (in Japanese).

4) Watanabe, Y., Nakano, M.: 5. Validation and Application of a Numerical Tool for Lifetime Evaluation of lon Thruster's lon Optics, J. Plasma Fusion Res., 90 (2014), pp. 252-260 (in Japanese).

5) Holland, H. J.: Adaptation in Natural and Artificial Systems, Bradford, Cambridge, 1975.

6) Beasley, D., Bull, R. D., and Martin, R. R.: An Overview of Genetic Algorithms Part 1, University Computing, 15 (1993), pp. 58-69.

7) Higuchi, T., Kitano, T.: Genetic Algorithms, IPSJ, 34 (1993), pp. 871-883 (in Japanese).

8) Morikita, H.: Genetic Algorithm, Morikita, Tokyo, 2002 (in Japanese).

9) Kitano, T.: Genetic Algorithms, JSAI, 7 (1991), pp. 26-37 (in Japanese).

10) De Jong, K. A.: Analysis of The Behavior of a Class of Genetic Adaptive Systems, Ph.D. Thesis, University of Michigan Ann Arbor, 1975.

11) Stender, J., Hillebrand, E., and Kingdon, J.: Genetic Algorithms in Optimization, Simulation and Modelling, IOS Press, Amsterdam, 1994.

12) Hanada, Y.: Genetic Algorithm, Engineering \& Technology, 15 (2008), pp. 13-19 (in Japanese).

13) Yamamoto, N., Kondo, S., Chikaoka, T., Masui, H., and Nakashima, H.: Effects of Magnetic Field Configuration on Thrust Performance in A Miniature Microwave Discharge Ion Thruster, J. applied physics, 102, 123304 (2007).

14) Goebel, M. D., Katz, I.: Fundamentals of Electric Propulsion: Ion and Hall Thrusters, John Wiley \& Sons, Hoboken, 2008, pp. 232-235.

15) Nakano, M.: Fast Calculation of Ion Machining for Ion Thruster Accelerator Grid, IAPC, 2 (2018), pp. 65-72 (in Japanese). 